\title{
Industrial Use of HiPIMS up to Now and a Glance into the Future, A Review by a Manufacturer Introduction of the hiP-V hiPlus Technology
}

\author{
Gerhard Eichenhofer ${ }^{1, *}$, Ivan Fernandez ${ }^{2}$, Ambiörn Wennberg ${ }^{3}$ \\ ${ }^{1}$ Department of Applied Physics, University of Applied Sciences Ravensburg-Weingarten, Germany \\ ${ }^{2}$ Institute of Microelectronics of Madrid, Spain \\ ${ }^{3}$ Royal Institute of Technology, Sweden
}

Copyright $\bigcirc 2017$ by authors, all rights reserved. Authors agree that this article remains permanently open access under the terms of the Creative Commons Attribution License 4.0 International License

\begin{abstract}
It has been demonstrated by several groups that HiPIMS is a state of the art tool for applying demanding coatings with superior film properties. The real industrial breakthrough for the HiPIMS-technology has not yet happened. On the other hand, the up till now available HiPIMS-PS were mainly been up-scaled "prototypes", far away from industrial work horses. With the hiP-V HiPIMS power system, a direct derivative of a robust power supply technology already in commercial use for public transportation systems, another milestone is set to make the HiPIMS technology go mainstream. HiPIMS is not a revolution that will make all other technologies obsolete, yet it is a very powerful complement. With a reliable, multi-functional power supply and with a rapid arc-handling, it could possibly be a start of a new era in thin film production. Just consider the possibility of etching and implantation to increase cleanliness and adhesion of the samples. Until now, most of the R\&D work done in HiPIMS, has been dedicated to hard coatings and tool coatings. Here, HiPIMS is surely useful but not the expected technological breakthrough. For the future, the implementation of the new pos. reverse pulse, the hiP-V hiPlus HiPIMS technology, is opening a whole new field of possible applications for i.e. nonconductive substrates where no bias can be applied. Glass and plastics can be processed with remarkable results in hardness, enhanced film properties and additionally, it is achieved at lower substrate temperatures. It has been a slow start for HiPIMS, but the future looks bright.
\end{abstract}

Keywords HiPIMS, HPPMS, Process, PVD, PECVD, Ionized Processing, Magnetron Sputtering Thin Film Deposition

\section{Introduction}

Thinking of DC, DC-Pulse and RF as classical magnetron sputtering technologies, HiPIMS (High Power Pulsed Magnetron Sputtering) can be considered the most recent and state of the art magnetron sputtering technique. The kick-off of the HiPIMS or HPPMS (High Power Pulse Magnetron Sputtering) technology was in 1999 by a paper from V. Kousnetzov [1]. The characteristic of this technology is that the power is applied in high energetic pulses with low duty cycle $(<0.1)$ and frequency $(<2 \mathrm{kHz})$. The pulse power densities on the target are several $\mathrm{kW} / \mathrm{cm}^{2}$. This mode of operation results in the creation of very dense plasmas which allows a high degree of ionization of the sputtered material. The result provides great benefits, such as: drastically reducing defects or macroparticles when compared with cathodic arc deposition, extremely dense films, high hardness, excellent adhesion and low surface roughness $[2,3]$. The films are more homogenous and less sensitive for complex shapes. Due to the presence of multiple charged ions, there is a possibility to implant sputtered atoms into the atom matrix, achieved when combined with a substrate Bias, of the base material. This further improves film adhesion and enables the possibility to manipulate the residual stresses in the surface of the object, reducing the risk for fatigue and corrosion related damage.

Since the introduction, many examples of successful coatings prepared by HiPIMS can be found in the literature. Some examples are hard $\mathrm{CrN}$ with enhanced corrosion resistance [4], high dense DLC [5], fine grained alfa-alumina deposited at low temperature [6], nanoscale multilayers for oxidation resistance [7], high density transparent conductive oxides [8] or $\mathrm{TiO}_{2}$ optical coatings with high refractive index [9].

Despite the great perspective and the positive forecasts for the HiPIMS-technology, given by scientists and technologist in the past decade, the real industrial breakthrough has not yet started. HiPIMS is still considered a niche technology. Partly this could be the case because the HiPIMS power supply technology up to now was not yet properly 
industrialized. Part of the explanation can be that HiPIMS power supplies were and still are more expensive than traditional power supplies. There were severe reliability issues in the past and in some cases the HiPIMS-PS have even been unsafe to use. The deposition rate of HiPIMS is still considered to be rather low compared with conventional magnetron sputtering and even more, if compared to cathodic arc-deposition. In most so called industrial HiPIMS coating machines the HiPIMS-technology is solely used as substrate pretreatment. A common setup in batch coaters and multi-magnetron systems, where HiPIMS is used, are, one or two (in very rare cases more) magnetrons equipped with HiPIMS while the other cathodes (2-6 per system) are equipped with UBM (unbalanced magnetron deposition). The actual film-deposition is mainly done via UBM, not with HiPIMS.

Another issue, positive or negative, is the complexity of use due to the large number of adjustable parameters. It is not only the HiPIMS power supply, which itself has more controllable parameters than any traditional power supply, what contributes to this great deposition technology. It is also the process regulation (monitoring), the magnetron system (magnetic configurations), the gas flow, the pumping speed, etc. However, the HiPIMS power supply which is the heart of the HiPIMS-Technology is definitely the most parameter-sensitive and parameter-versatile processing instrument.

\section{The hiP-V Technology}

Due to the known shortcomings of the current HiPIMS products in the market, the motivation of the creators of the
hiP-V HiPIMS-PS is to deliver a highly reliable and multi-functional state of the art HiPIMS-power supply to the market, i.e. a power supply where HiPIMS can be used reliably. Also in combination or in addition to more conventional technologies, to use the most cost effective technology in each step of the process without the need of several power sources, and a power supply that is able to do so during continuous production without failing.

The general demand for HiPIMS-power supplies is: power systems which can deliver high power pulses, with a certain voltage / current / pulse length / frequency and have the ability to suppress arcs. This is in principle the same demand as for certain industrial electrical switch mode power supplies used in trains and subways. To guarantee the same high reliability and durability which is obligatory for public transportation systems, the hiP-V HiPIMS power supplies are built with the same subassemblies on an identical electrical power supply platform.

Designed as a multi-purpose "All in One" system, the hiP-V HiPIMS-PS can be used for multiple applications as a HiPIMS-PS, a HiPIMS Bias-PS and as a DC/DC-pulsed PS. Unipolar or Bipolar processing as well as superimposed or sequential HiPIMS (in combination with DC or MF) is possible. All this, for single or dual magnetron applications.

The hiP-V products are unique for HiPIMS power supplies due to the high modularity and flexibility. The hiP-V power supplies can be connected in parallel to increase the power up to hundreds of $\mathrm{kW}$ or the current to be in the two digits' kA-range. This, the wide power range and the fast Arc-handling make the hiP-V product line dedicated for high volume industrial production at a reasonable cost.
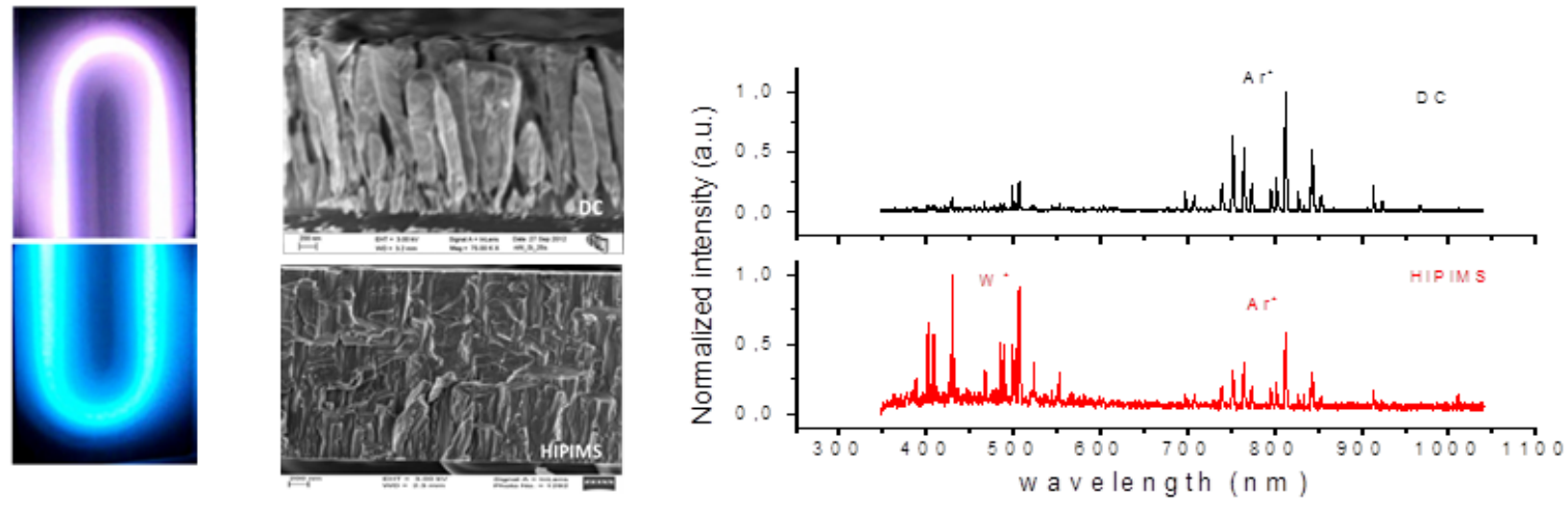

Figure 1. Left: Picture of DC plasma, top, vs HiPIMS plasma, bottom. Both established in an industrial magnetron. Center: Cross-sectional SEM captions of tungsten coatings deposited by straight DC and HiPIMS (credits: Nano4Energy SL). One can observe the more dense packed film structure with HiPIMS-deposition. Right: OES Spectrum DC vs HiPIMS. 

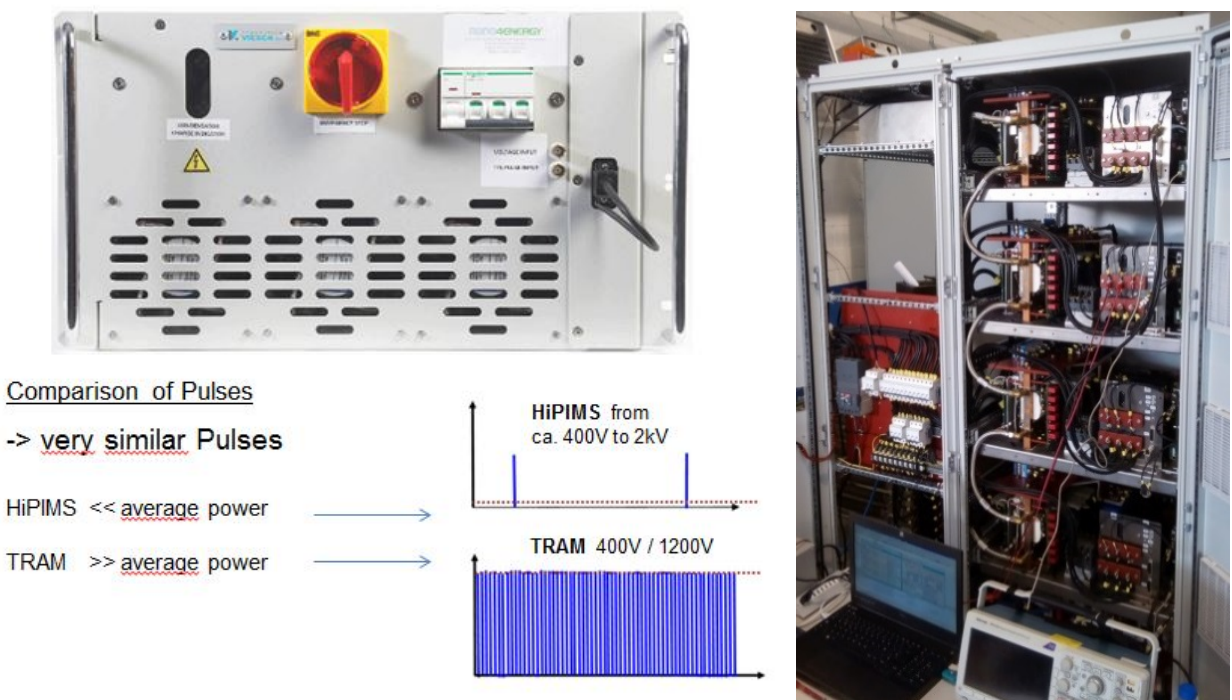

Figure 2. Left, Picture of the $6 \mathrm{~kW}$ hiP-V HiPIMS power supply. Schematics of a pulsed power supply operation in both plasma and train environments. Right, Picture of an $80 \mathrm{~kW}$ hiP-V HiPIMS power pack. This cabinet consists in four modules of 20kW each, one module can be operated as Bias HIPIMS, while the other three can be synchronized and operated in parallel to multiply peak current and average power values.

\subsection{HiPIMS Power Pack}

The "All in One" functionality of the hiP-V HiPIMS PS makes it ideal to be combined in a HiPIMS PS power pack. As shown in Figure 2, this HiPIMS PS power pack is a stacked combination for several hiP-V units, to multiply the power and current output. Of course, it is still possible to use the individual unit in single operation. A high-power switch makes it possible to combine the selected outputs of the HiPIMS-PS and leads the process power to the designated magnetron or to the substrate as a Bias. An 80KW HiPIMS power pack, consisting of $4 \times 20 \mathrm{KW}$, is already industrialized. The power pack technology enables coatings for substrate sizes of up to several meters.

\subsection{Arc Management}

This subject has been neglected at the very beginning of the HiPIMS technology. It was said that HiPIMS does not need to have Arc handling. However, it has been proven in an early stage that arc handling is of utmost importance. Due to the immense power density delivered to the target surface, the damages of uncontrolled arcs are extremely severe and not at all negligible. Even more, in high voltage Bias applications, such as for HiPIMS metal etching, it is crucial to achieve reproducible metal etching rates, implantation depths, and prevent arc damages at the substrate surface. Once the arc is detected on the substrate or at the magnetron, all involved power systems must immediately enable the ARC-handling and stop the energy supplied into the hot spot as soon as possible (normally $<3$ us).

A new feature in the hiP-V product range is the possibility to apply a positive voltage reversal called the "hiPlus" at the end of the negative pulse. This approach has already been tested in asymmetric bipolar pulsed magnetron sputtering [10], resulting very effective to reduce the probability of arcs

\section{to appear.}

The hiP-V product line demonstrates state of the art Arc-handling behavior [11]. The arc can be detected via absolute value or delta values, i.e. the classic current detection or, the more arc sensitive $\mathrm{dI} / \mathrm{dU}$ voltage mode detection which is new for HiPIMS-PS. The arc detection voltage threshold value can be set in percentage of the actual voltage drop. A voltage drop is immediately evident, when an arc begins to form. As soon as the voltage drop occurs, the output power is suppressed and the voltage on the output of the HiPIMS-PS is reversed, as shown in Figure 3. This positive voltage reversal recovers or removes the stored energy in the cable, the magnetron and last but not least, the plasma, i.e. no energy will continue to feed the initiated arc event.

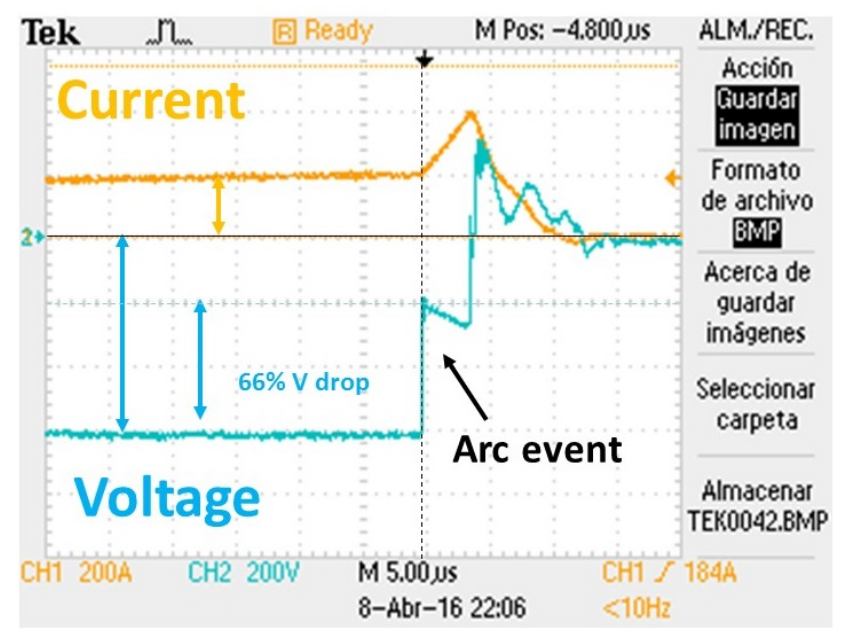

Figure 3. Oscilloscope Caption of Voltage and Current signals during an arc event. Notice the voltage drop and the current raise, until the arc protection circuitry actuates and reverses the polarity. 


\section{Classical Applications, Industrial Use of HiPIMS up Until Now}

\subsection{HiPIMS Metal Etching Pre-treatment}

Looking at the great film properties achieved with HiPIMS technology, up until now, the true value of HiPIMS in the industry has come to life in pretreatments prior to hard coating deposition, such as substrate metal ion etching and implantation. Bombardment with metal ions is known to provide cleaner interfaces and effective etching of the substrate oxide layers. This was the base of the development in the 1990's of the so-called ABS (Arc Bond Sputtering) technique [12]. The described technique uses a cathodic arc source to generate metal ions that are accelerated by a DC Bias voltage towards the substrate to produce an etching prior to the film deposition by magnetron sputtering. The high flux of metal ions not only clean the substrate, but also produce low-energy implantation, (of up to $15 \mathrm{~nm}$ ) generating an engineered interface gradient and enhancing adhesion through local epitaxy of the coating on the substrate. A drawback of this technique is the production of droplets or macroparticles during the metal etching process that are deposited onto the sample surface, thus creating a large-scale coating defect as the thickness increases [13], shown in
Figure 4. In 2003 the idea was adapted to the HiPIMS technology [14]. The substrate metal etching was performed using a $\mathrm{Cr}$ magnetron working in HiPIMS mode, which allows the presence of single and double charge $\mathrm{Cr}^{+}$metal ions. Two HiPIMS power supplies were used in a masterslave configuration, one to establish the plasma discharge in the magnetron and the second to withstand the high bias voltage during the current pulse arriving to the substrate. Better adhesion figures were achieved as compared with ABS, and without the presence of droplets or molten macroparticles. Clean interfaces and large areas of epitaxial growth were initially published for coatings such as $\mathrm{CrN}$ or TiAlN [15]. This substrate pre-treatment technique was also used for other coatings such as carbonitrides CNx [16], or carbon compounds: WC $[17,18]$ or DLC $[19,20]$.

Most recently, the presence of multiply charged metal ions such as $\mathrm{Ti}^{+4}$ [21] has been demonstrated in HiPIMS discharges. This finding required a Ti HiPIMS discharge at high magnetron current densities, up to $5 \mathrm{~A} / \mathrm{cm}^{2}$, but is very relevant for the industrial processing, as it would allow the reduction of applied bias voltage during HiPIMS metal etching, thus, minimize the appearance of unwanted arcs on the substrate [20].
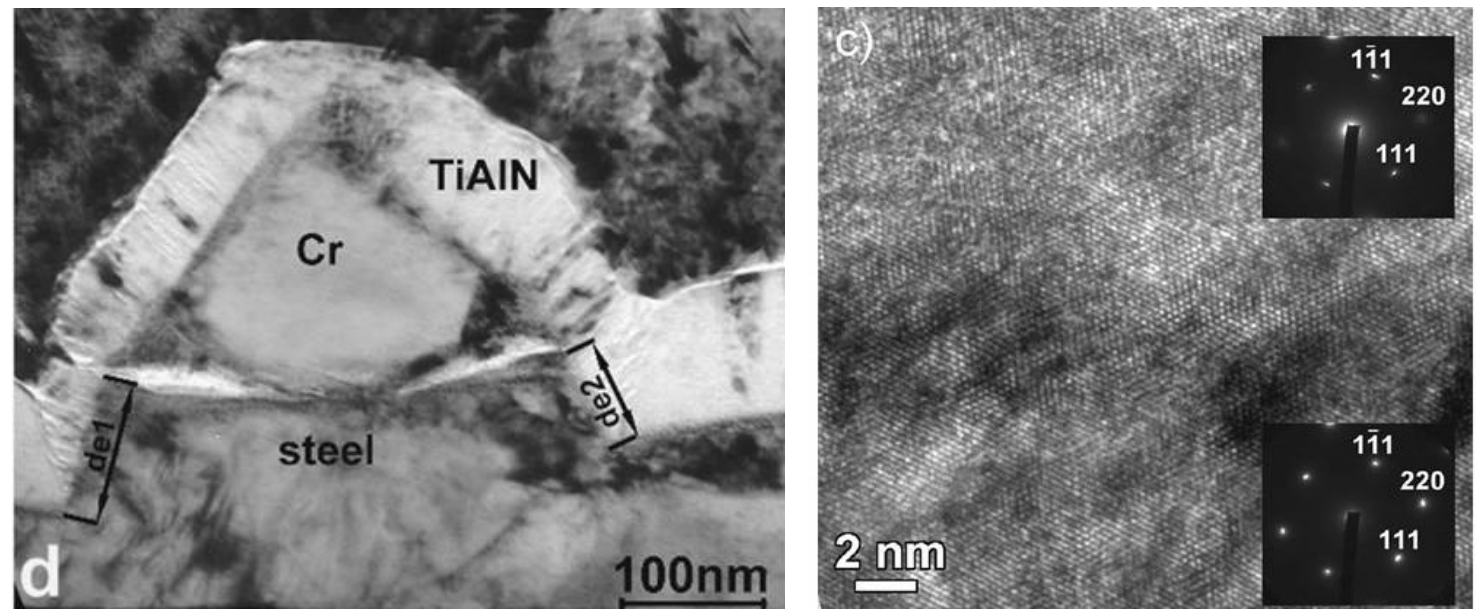

Figure 4. Left, the presence of an unwanted droplet in arc $\mathrm{Cr}$ etch using a cathodic arc is used to show evidence of substrate material removal of $100 \mathrm{~nm}$ [Data taken from Ref. 13]. Right, epitaxial CrAlN layer grown on the gamma-TiAl substrate after HiPIMS Cr pretreatment. Uniform diffraction contrast is shown [Data taken from Ref. 15]. 

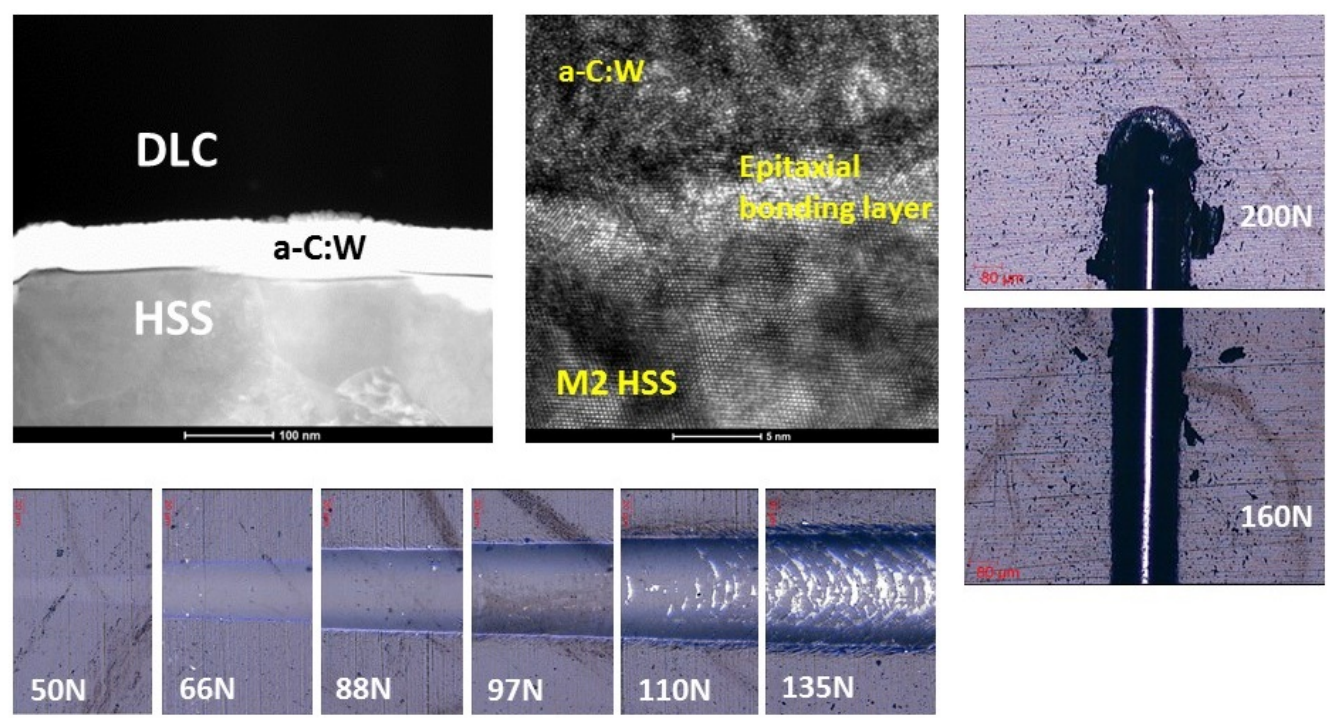

Figure 5. HRTEM image of a WC-DLC coating deposited onto M2 HSS with HiPIMS metal etching. The scratch measurements show excellent coating adhesion.
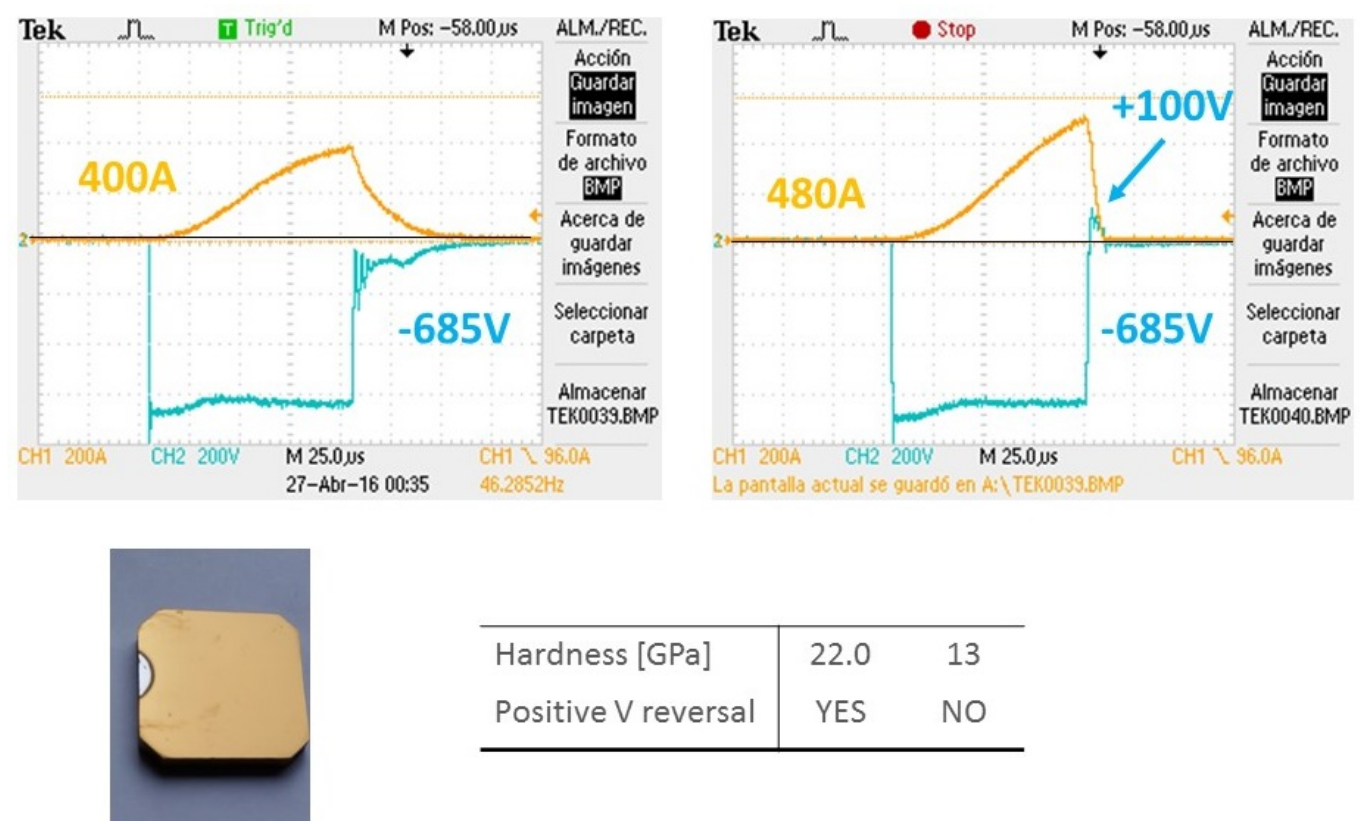

\begin{tabular}{l|cc}
\hline Hardness [GPa] & 22.0 & 13 \\
Positive V reversal & YES & NO \\
\hline
\end{tabular}

Figure 6. Oscilloscope captions of Voltage and Current signals during reactive Ti HiPIMS plasma in the presence of Argon and Nitrogen. Both plasmas were run at the same constant voltage $(685 \mathrm{~V})$, pulse Time ON (100us) and frequency values $(300 \mathrm{~Hz})$ but with [Left] and without [Right] a positive voltage reversal at the end of the pulse. Notice the faster current extinguish with the voltage reversal and the higher peak current values at the same. The output average power is in the range of $3 \mathrm{~kW}$.

\section{Next Generation HiPIMS: Positive Voltage Reversal for Positive Ion Assisted Deposition on Insulating Substrates with the hiP-V, hiPlus Option}

Some perspectives to further increase the positive ion bombardment towards the substrate and perhaps the deposition rate was envisaged during the last years. This new approach called "hiPlus", will apply a reverse positive voltage pulse to the cathode, after the primary plasma-ON sputtering pulse, thus accelerating the positive ions present in the plasma. The right combination of voltage reversal with the magnetron magnetic design can enhance ion assistance to the depositing film without applying a bias voltage, thus, tailor coating properties. From the technological point of view, these results are very interesting as it allows the ion assisted deposition of coatings onto insulating substrates, such as polymers, glass or textiles, where no Bias voltage can be applied.

The presence of high-energy ions in asymmetric pulsed bipolar DC discharges with un-controlled positive voltage overshoot has already been measured using energy resolved 
mass spectroscopy. Higher ion energies per deposited atom were demonstrated for the deposition of dense oxide films [22] as well as DLC coatings with hardness values up to $32 \mathrm{GPa}$ [20]. The presence of a short positive pulse, during the plasma OFF sequence in the pulse, discharge the magnetron and substrate surface, and thus, reducing the tendency to arcing. By not using a bias, it is also possible to avoid charge buildup on the substrate which often leads to inhomogeneous coatings with high residual stresses.

For this purpose, the team behind hiP-V is developing a new pulsing mode where the appearance of positive voltage reversal is active and controllable to maximize the effect with or without bias. Some oscilloscope captions are shown in Figure 6 for a reactive Titanium Nitride deposition, from TiN coatings deposited at RT onto Si and M2 HSS substrates, without the application of Bias. The hardness values were obtained with a nanoindenter. The result was $22 \mathrm{GPa}$ when the voltage reversal was applied, considerably higher than the value without the application of the positive voltage reversal, $13 \mathrm{GPa}$.

\section{New Possibility for Applications}

Besides the well-known advantages of highly ionized deposition already reported, there are obviously many more interesting applications that appear on the screen every time scientists and engineers sit together and talk about the HiPIMS technology. The handicap for the implementation of these ideas into the consumers' world is unfortunately still the high cost vs. the proven benefit on the final product. Nevertheless, the HiPIMS evolution will continue to develop with all its advantages. The shortcomings are rapidly disappearing one by one, faster deposition rates, lower production costs and more reliable process equipment are already on the verge of entering the production lines. This technology will finally take its place were the forecasts of scientists and technologist expected it to be already in the last decade.

The temperature delivered to the substrate is significantly lower for HiPIMS than compared to DC or DC-Pulse magnetron sputtering [9]. This technology opens a promising future for new applications of coatings on temperature sensitive substrates, such as plastics, textiles and any other materials were a low temperature deposition is crucial.

Among other fields, outside the hard and decorative coatings the technology can be applied in functional applications such as, long lasting antibacterial coatings of medical materials via HiPIMS, e.g. medical linens, surgical instruments, partition walls or even door knobs and handles for water faucets in sensitive amicrobic areas. Similar HiPIMS technologies but one step further into consumers' applications is the treatment of high-end sports- and functional-wear, where the life time and function of the garments can be greatly increased. Also in the food packaging sector, various barrier coatings of foils and wraps can be treated via the HiPIMS technology to enhance the performance.

\section{Summary}

It has been demonstrated by several groups that HiPIMS is a state of the art tool for applying demanding metal, DLC and ceramic coatings with superior properties for present and future applications. Nevertheless, the real industrial breakthrough for the HiPIMS-technology has not yet happened. On the other hand, the up till now available HiPIMS-PS was anything but true industrial work horses. The power supplies have mainly been "prototypes" that were up-scaled and put into service in the industry. With the truly reliable and purpose built hiP-V HiPIMS power system, a direct derivative of a robust power supply technology already developed and in commercial use for public transportation systems throughout the world, another milestone is set to make the HiPIMS technology go main stream.

Seeing the technology with a critical eye, the HiPIMS is not a revolution that will make all other technologies obsolete. However, it is a very powerful complement. With a reliable power supply that can switch between different power options and with a ultra-fast arc-handling, it could possibly be a start of a new era in thin film production. As a start, consider the possibility of etching and implantation to increase cleanliness and adhesion of the samples.

Until now, most of the R\&D work done in HiPIMS, has been dedicated to hard coatings and tool coatings. Here, HiPIMS is surely useful but not the expected technological breakthrough. For the future, the implementation of the new positive reverse pulse, the hiP-V hiPlus HiPIMS technology, is opening a whole new field of possible applications for i.e. nonconductive substrates where no bias can be applied. Glass and plastics can be processed with remarkable results in hardness, enhanced film properties and additionally, it is achieved at lower substrate temperatures.

It has been a slow start for the HiPIMS, but the future looks bright.

\section{REFERENCES}

[1] V. Kouznetsov, K. Macak, J.M. Schneider, U. Helmerson and I. Petrov: Surf. Coat. Technol. 122 (1999) 290-293 “A novel pulsed magnetron sputter technique utilizing very high target power densities".

[2] K Bobzin, N Bagcivan, P Immich, S Bolz, J Alami, R Cremer J. Mater. Process. Technol. 209165 (2008). "Advances of nanocomposite coatings deposited by HIPIMS"

[3] K. Sarakinos, J. Alami, S. Konstantinidis Surface and Coatings Technology Volume 204, Issue 11 (2010), 16611684 "High power pulsed magnetron sputtering: A review on scientific and engineering state of the art". 
[4] Reinhard, C.Ehiasarian, A.P.; Hovsepian, P.Eh.: Thin Solid Films, v 515, n 7-8, Feb 26, 2007, p3685-3692 “CrN/NbN superlattice structured coatings with enhanced corrosion resistance achieved by high power impulse magnetron sputtering interface pre-treatment".

[5] Asim Aijaz, Kostas Sarakinos, Daniel Lundin, Nils Brenning, Ulf Helmersson. Diamond and Related Materials, 23, 1-4 (2012) "A strategy for increased carbon ionization in magnetron sputtering discharges".

[6] E. Wallin, T. I. Selinder, M. Elfwing and U. Helmersson. Europhysics Letters, Volume 82, Number 3 "Synthesis of $\alpha$-Al2 O3 thin films using reactive high-power impulse magnetron sputtering".

[7] P. E. Hovsepian, C. Reinhard, A. P. Ehiasarian. Surf. Coat. Technol. 201, 4105 (2006) "CrAlYN/CrN superlattice coatings deposited by the combined high power impulse magnetron sputtering/unbalanced magnetron sputtering technique".

[8] V. Sittinger, F. Ruske, W. Werner, C. Jacobs, B. Szyszka, D.J. Christie, Thin Solid Films 516 (2008) 5847. "High power pulsed magnetron sputtering of transparent conducting oxides".

[9] S. Konstantinidis, Thin Solid Films 515 (2006) 1182. "Titanium oxide thin films deposited by high-power impulse magnetron sputtering”.

[10] J. Sellers, Surf. Coat. Technol. 98 (1998) 1245 “Asymmetric bipolar pulsed DC: the enabling technology for reactive PVD”.

[11] A. Anders, Thin Solid Films 502 (2006) 22 - 28 "Physics of arcing, and implications to sputter deposition".

[12] G. Hakansson, L. Hultman, J.-E. Sundgren, J. E. Greene, and W.-D.Muenz, Surf. Coat. Technol. 48, 1 (1991) "Microstructures of TiN films grown by various physical vapour deposition techniques".

[13] C. Schonjahn, L. A. Donohue, D. B. Lewis, W.-D. Munz, R. D. Twesten, and I. Petrov, J. Vac. Sci. Technol. A 18, 4 (2000) "Enhanced adhesion through local epitaxy of transition-metal nitride coatings on ferritic steel promoted by metal ion etching in a combined cathodic arc/unbalanced magnetron deposition system".
[14] A.P. Ehiasarian, W.-D. Muenz, L. Hultman, U. Helmersson, I. Petrov, Surface and Coatings Technology 163 -164 (2003) 267-272 "High power pulsed magnetron sputtered CrNx films".

[15] A. P. Ehiasarian, J. G. Wen and I. Petrov. Journal of Applied Physics 101, 054301(2007) "Interface microstructure engineering by high power impulse magnetron sputtering for the enhancement of adhesion".

[16] E. Broitman, Zs. Czigány, G. Greczynski, J. Böhlmark, R. Cremer, L. Hultman Surface \& Coatings Technology 204 (2010) 3349-3357 "Industrial-scale deposition of highly adherent CNx films on steel substrates".

[17] Martin Drábik, Vladimír Ballo, Martin Truchlý, Juraj Frkáň, Tomáš Roch, Lenka Kvetková, Leonid Satrapinskyy, Peter Kúš, Surface \& Coatings Technology 293 (2016) 2-9 "Influence of plasma pretreatment on the performance of industrial tungsten carbide coatings deposited at low temperature on 100Cr6 bearing steel substrates".

[18] Wolf-Dieter Münz Galvanotechnik 4774 (2016) "Superharte C-DLC-Schichten abgeschieden mittels HIPIMS/UBM-Kathodenzerstäubung”.

[19] L.A. Donohue, A. Torosyan, P. May, D.E. Wolfe, J. Kulikand T.J. Eden. Plating \& Surface Finishing, March 200938 "Investigation of PVD-DLC Thin Films Manufactured Using HIPIMS Etch / Unbalanced Magnetron Sputter (UBM) Deposition and Secondary Mechano-Chemical Modification".

[20] Ivan Fernández.-Martinez, Victor Bellido-Gonzalez, Jose Antonio Santiago-Varela, Raquel Gonzalez-Arrabal, Ambiorn Wennberg, Frank Papa, Miguel Monclús and Jon Molina, Technical Conference Proceedings 2016 Society of Vacuum Coaters. "Tribo-mechanical properties of hard hydrogen-free DLC coatings prepared by magnetron sputtering”.

[21] Joakim Andersson, Arutiun P. Ehiasarian, and André Anders, Applied Physics Letters 93, 071504 (2008) “Observation of Ti $4+$ ions in an high power impulse magnetron sputtering plasma".

[22] J W Bradley, H Bäcker, Y Aranda-Gonzalvo, P J Kelly and R D Arnell, Plasma Sources Science and Technology, Volume 11,2 "The distribution of ion energies at the substrate in an asymmetric bipolar pulsed DC magnetron discharge". 